\title{
Adaptive infill sampling strategy for metamodeling: Challenge and future research directions
}

\author{
Che Munira Che Razali ${ }^{1}$, Shahrum Shah Abdullah ${ }^{2}$, Amir Parnianifard ${ }^{3}$, Amrul Faruq $^{4}$ \\ ${ }^{1,2,4}$ Department of Electronic System Engineering, Malaysia-Japan International Institute of Technology, \\ Universiti Teknologi Malaysia \\ ${ }^{3}$ Department of Mechanical and Manufacturing Engineering, Faculty of Engineering, Universiti Putra Malaysia
}

\begin{tabular}{l} 
Article Info \\
\hline Article history: \\
Received Dec 23, 2019 \\
Revised Mar 3, 2020 \\
Accepted Apr 9, 2020 \\
\hline
\end{tabular}

Keywords:

Adaptive sampling

Metamodel

Optimization

\begin{abstract}
The widespread use of computer experiments for design optimization has made the issue of reducing computational cost, improving accuracy, removing the "curse of dimensionality" and avoiding expensive function approximation becoming even more important. Metamodeling also known as surrogate modeling, can approximate the actual simulation model allowing for much faster execution time thus becoming a useful method to mitigate these problems. There are two (2) well-known metamodeling techniques which is kriging and radial basis function (RBF) discussed in this paper based on widely used algorithm tool from previous work in modern engineering design of optimization. An integral part of metamodeling is in the method to sample new data from the actual simulation model. Sampling new data for metamodeling requires finding the location (or value) of one or more new data such that the accuracy of the metamodel can be increased as much as possible after the sampling process. This paper discussed the challenges of adaptive sampling in metamodel and proposed an ensemble non-homogeneous method for best model voting to obtain new sample points.
\end{abstract}

This is an open access article under the CC BY-SA license.

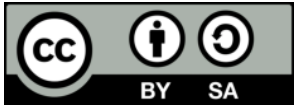

\section{Corresponding Author:}

Shahrum Shah Abdullah,

Department of Electronic System Engineering,

Malaysia-Japan International Institute of Technology,

Universiti Teknologi Malaysia, Jalan Sultan Yahya Petra, 54100 Kuala Lumpur, Malaysia.

Email: shahrum@utm.my

\section{INTRODUCTION}

Computer simulation is ubiquitous in engineering design and optimization to solve the complex system. Metamodeling is known as surrogate modeling or "model of the model" delivers significantly better performance for solving a complex system to mitigate computational cost, improve accuracy, and find the optimal solution in optimization. Typical types of surrogate modeling that recently used by the researcher is polynomial response surface (PRS), kriging or Gaussian process, radial basis function (RBF), support vector regression (SVR), multivariate spline (MARS), and artificial neural network (ANN). Each model of surrogate modeling has its advantages and disadvantages depends on the complex function and optimization model. Optimization is the method of finding the optimum objective function value or cost function $f(x)$ based onthe input variable value given by $\mathrm{x}$. Without using metamodeling, an approximation of function $\mathrm{f}(\mathrm{x})$ might require a higher number of times for function approximation to find the optimal value of variables in cost function $f(x)$. Hence, recent studies from the previous author emphasized that the advantages of metamodeling are the reduction of the computational cost [1-4]. 
Current trends in metamodeling have led to a proliferation of studies that able to predict the value of the objective function and constraints accurately at a new design point without repetition original expensive simulation [5]. Metamodeling based optimization techniques consist of two stages: i) initial design using the design of experiment ii) approximation function using metamodeling. In an investigation of metamodel techniques, Huachao et al. [6] state that there is another two-step in metamodeling optimization (i) Exploit surrogate models to capture the potential optimal points as added samples; (ii) Explore the sparsely sampled regions to guarantee a balance between global and local search. The steps in metamodel similar to those reported by several previous researchers for exploration and exploitation design space [7-9]. Metamodel method in the global optimization algorithm can provide a better solution to achieve convergence, improve accuracy, robust, and can explore the region without stuck in local valleys.

A large and growing body of literature study investigated the fact that the application of a metamodel in the engineering field agreed that this method provides an optimal solution quickly than any other algorithm [10-15]. Review for kriging metamodeling publishes by [16] to present basic kriging formula and extend kriging simulation using bootstrap to estimate the variance of kriging predictor. Another work also published by the same author review kriging metamodel in experimental design and proposed "robust" optimization accounts for uncertainty in some simulation input with the Taguchi method. The existing metamodeling review extensively and focuses mainly in engineering optimization problem such as complexity of crashworthiness problem [17], simulation complex system of aircraft and aerodynamic design [18], architecture design of optimization [3], simulation-based complex engineering design and employing surrogate model in robust model optimization (RBO) framework [19]. In short, much of prior metamodel studies concentrated on approximate function, finding the location of the observed point to improve metamodel accuracy and explore and exploit the region for uncertainty analysis.

Selection of model in surrogate model optimization is difference based on test function approximation and constraint handling. Khairy et al. (2012) investigate comparison two (2) metamodel techniques, RBF and Kriging in different aspects such as accuracy, robustness, efficiency, and scalability to identify advantages and disadvantages of each meta-modeling technique. His study found that the RBF metamodel is more accurate than the kriging metamodel. Another study compared PRS with RBF for experimental work demonstrated that computational simulation adopted RBF gets a better result and suggested to enhance the number of level in design space to increase the accuracy of metamodel [20]. Østergård's et al. Comparative study (2018) found that that Kriging produces the most accurate metamodels, followed by ANN and MARS but becomes inefficient for large training sets. This author compares six (6) major metamodeling techniques such as linear regression with ordinary least squares (OLS), random forest (RF), support vector regression (SVR), multivariate adaptive regression splines (MARS), Gaussian process regression (GPR), and neural network $(\mathrm{NN})$. The comparison method concerning accuracy, efficiency, easeof-use, robustness, and interpretability for overall metamodel [21]. Selection of metamodel approximation is depend on input parameter, number of samples and complexity of the system. Likewise, RBF metamodel widely used to predict time series, control parameter of control system and data mining [22, 23].

Unlike the comparison of six metamodels, comparative research between kriging and RBF metamodeling techniques for design optimization of variable stiffness composites indicates that both models are the most precise and robust model in design space exploration. This author showed that Kriging's suitability is good for a small number of design variables, while RBF metamodel is the best model for a large number of variables [24]. Previous research in 2016 by Vicario compared kriging and ANN metamodel in computer experiment indicate that predictive using kriging is acceptable unfortunately the model not satisfy the predictive accuracy while ANN also gives acceptable result, but it is hard to understand their inner workings [25]. In his case study of the effect, Latin Hypercube sampling on metamodel shows that RBF showed the best overall performance in global exploration when comparing to Kriging. For future research work, this author suggested further improvement in exploration or exploitation characteristics can be assisted using 'adaptive sampling' strategies [26]. The evidence reviewed here seems the studies of metamodel optimization technique indicate that there no best model suitable for all mathematical function approximation. The metamodel employed in optimization based on design function, variable, dimension, and constraint handling.

Therefore, the advantages of using metamodel in optimization problem able to reduce cost and improved accuracy. However, not every model of metamodel suitable for all problem in design optimization because difference metamodel is shown to perform well indifference problem and condition. On the contrary previous research work in metamodeling, ensemble method is another way to fit one or more metamodel and select the one metamodel that performs best. The first work adopts an ensemble method in metamodel proposed by Goel et al. in 2007, implemented a weighted average based on an error in each metamodel to choose the best metamodel [27]. Zhou (2016) in his research implement ensemble method to select suitable metamodel for objective-oriented sequential sampling proposed genetic algorithm (GA) to optimal weight 
coefficient for each metamodel [28]. Genetic-based algorithm is a sort of systematic random search by imitating evolutionary processes in nature. GA is a technique of combining one chromosome with the finest chromosomes in the current population [29, 30]. Latest work by Li et al. in 2019 proposed ensemble of surrogates assisted particle swarm optimization (EAPSO) algorithm for medium scale expensive problems to enhance surrogate-assisted evolutionary algorithm because it trapped in local optima [31]. Base on analysis of previous research work, new future research direction can be highlighted to address issue adaptive sampling in engineering design.

Based on a comprehensive review, the main objective of this paper to propose a new approach of implementing an ensemble method in adaptive sampling stage to enhance sampling technique in order to improve metamodel approximation. Instead of implement ensemble method to choose the best metamodel, we attempt to propose the method implementing consensus or non-homogeneous ensemble method to voting over the best model in sampling new sample. To the best author knowledge, there is no research work implementing consensus in adaptive sampling strategy. The contribution of this paper is: (i) proposed method of infill sampling criteria which include deterministic and metaheuristic method for new sample selection to improve accuracy of metamodel, (ii) employ non-homogenous ensemble method for model voting the best location of new sample.

\section{TYPES OF METAMODELING}

The fundamental of metamodel is comes from response surface methodology (RSM) and this method strongly related to design of experiment to reduce number of simulation or experiment. In this section, only two types of metamodel Kriging and RBF discussed based on suggested by previous literature. The development of Kriging metamodel based on statistical method proposed by Daniel Gerhardys Kridge. This method purposely developed by expert in the geostatistic field for prediction and suitable for nonlinear problem [32]. RBF proposed by Hardy in 1971 designed to handle multivariate data interpolation [33]. $\mathrm{RBF}$ is one of the algorithm that works well in noisy data, suitable in function approximation, prediction time series, and fast convergence [34].

\subsection{Kriging}

For design input sample points $X=\left[x_{1}, \ldots, x_{m}\right]^{N}$, ( $\mathrm{m}$ is the number of sample and $\mathrm{N}$ is the number of variables) where $X \in R^{m x n}$ and the output response $Y=\left[Y_{1}, \ldots, Y_{m}\right]^{N}$ with $Y \in R^{m x n}$, kriging model is the combination of the trend term and the deviation term as (1),

$$
\hat{y}(x)=f(x)+Z(x)
$$

where $\hat{y}(x)$ is the predicted value of the kriging model, $\mathrm{f}(\mathrm{x})$ is the real function of $\mathrm{x}, \mathrm{z}(\mathrm{x})$ is a random process that provides global optimization model in design space similar to PRS. The covariance formula can be expressed as (2),

$$
\operatorname{cov}\left[Z(x)^{i}, Z(x)^{j}\right]=\sigma R\left[R\left(x^{i}, x^{j}\right]\right.
$$

$\mathrm{R}$ is the correlation matrix, $\mathrm{R}\left(\mathrm{X}^{\mathrm{i}}\right.$ and $\left.\mathrm{X}^{\mathrm{j}}\right)$ is the correlation function of any two samples points $\mathrm{X}^{\mathrm{i}}$ and $\mathrm{X}^{\mathrm{j}}$. Kriging metamodel has correlation function such as exponential function, Gaussian function, spline function.

\subsection{Radial basis function}

Given vector of input which $\mathrm{N}$ design space $\left[\mathrm{X}_{1}, \mathrm{X}_{2}, \ldots, \mathrm{X}_{\mathrm{N}}\right]$ and its corresponding responses $\mathrm{y}=\left[\mathrm{Y}_{1}, \mathrm{Y}_{2}, \ldots, \mathrm{Y}_{\mathrm{N}}\right]$, an RBF predictor at any point $\mathrm{X}$ in design space is given by (3),

$$
\hat{y}=\hat{f}(x)=\sum_{i=1}^{N} \omega_{i} \varphi\left(\left\|x-x_{i}\right\|\right)
$$

Where $\omega_{\mathrm{i}}$ is the weight coefficient evaluated by fitting the model to the training data; $\varphi($.$) is$ the nonlinear basis function, $\|$.$\| denotes the Euclidean distance between two sample points and \hat{y}$ is the predicted output of the objective function. The basis function weights, $\omega_{i}$, can be computed by using (4). The equation can write as below

$$
y=\varphi \omega
$$

The optimum value in the second layer weight computed using the least square formula as (5), 


$$
\widehat{w}=\left[\begin{array}{c}
w_{1} \\
w_{2} \\
\vdots \\
w_{N}
\end{array}\right]=\left(\varphi^{T} \varphi\right)^{-1} \varphi^{T}\left[\begin{array}{c}
Y_{1} \\
Y_{2} \\
\vdots \\
Y_{N}
\end{array}\right]
$$

Denoted that $y$ represented the vector of function values of training data, $\omega$ is the vector of basis function weight and $\varphi$ is the matrix design variables defined by (6),

$$
\varphi=\left[\begin{array}{cccc}
\varphi\left(x_{1}, x_{1}\right) & \varphi\left(x_{1}, x_{2}\right) & \ldots & \varphi\left(x_{1}, x_{N}\right) \\
\varphi\left(x_{2}, x_{1}\right) & \varphi\left(x_{2}, x_{2}\right) & \cdots & \varphi\left(x_{2}, x_{N}\right) \\
\vdots & \vdots & \ddots & \vdots \\
\varphi\left(x_{k}, x_{1}\right) & \varphi\left(x_{1}, x_{1}\right) & \ldots & \varphi\left(x_{k}, x_{N}\right)
\end{array}\right]
$$

Where $\mathrm{k}$ represented as designed variable and $\mathrm{N}$ is the number of the sample points. A typical radial basis function is the gaussian expressed by the below (7),

$$
\varphi_{i}(x)=e^{\left(-\frac{(x-c)}{\beta^{2}}\right)}
$$

Where $\mathrm{X}$ is the input data, $\mathrm{C}$ is the center and $\beta$ is spread parameter.

\section{ADAPTIVE SAMPLING USING METAMODEL OPTIMIZATION METHOD}

Metamodeling is a computational optimization technique using 'cheap to run model' which involve 4 stages: i) sampling technique ii) approximation function and iii) obtain new sample and iv) refining the metamodel. Simpson et al summarize used of each metamodel and fitting alternatives. RSM is established, easy to used and suitable for low dimension. Neural Network metamodel is best for high nonlinear problem huge samples and recommended for deterministic application. Kriging metamodel suitable for low dimension and flexible [10].

The framework is shown in Figure 1 which highlights the basic process of metamodeling-based optimization. First stage is sampling technique using design of experiment method. Second stage is choosing suitable metamodel method. Third stage is identified infill sampling or adaptive sampling method based on metamodel method in approximation stage. If the algorithm does not achieve stopping criteria or required sample points, add new sample point and repeat approximation stage until algorithm meets the number of samples needed. Finally, compute the validation error such as root mean square error (RMSE) to determine accuracy or performance measure of algorithm.

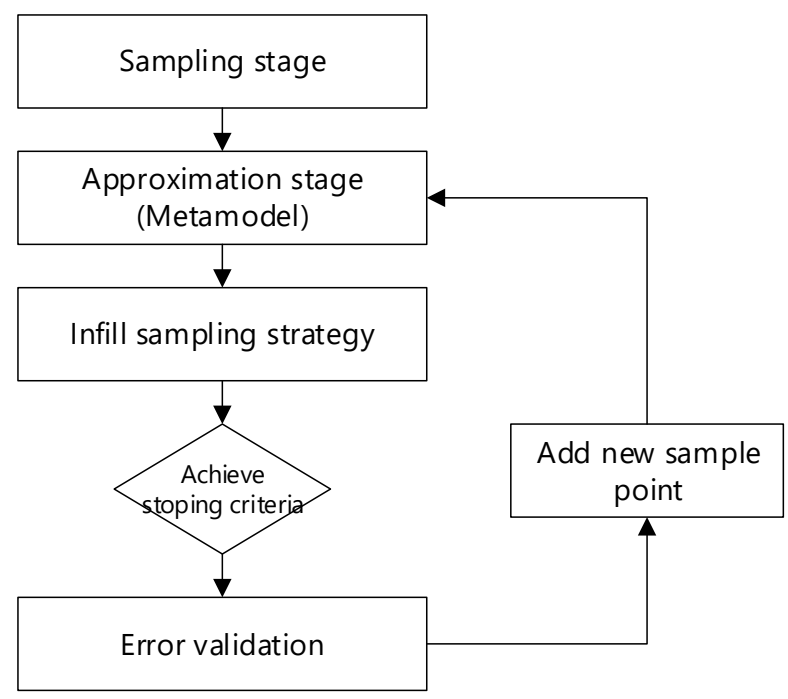

Figure 1. Metamodel-based optimization framework 


\subsection{Design of experiment}

Design of experiment (DoE) is the method to plan and reduce the number of experiments by control parameter of variables. DoE method introduced by Myers and Montgomery for the best sampling technique to reduce time and cost of real experiments. In metamodel based optimization, DoE method can improve the accuracy and mitigate the validation of error. Hence, the accuracy of metamodeling significantly relies on method of choosing initial sample points. Good experimental design selections can guide to identify inadequacies in the suggested models and prevent bias in the model. A set of the starting point of the entire domain is called design space. DoE is used as an effective method to solve computational simulation in Metamodel base optimization. DOE is a science that explores the most effective way of structuring the tests in order to obtain the maximum information by analyzing the test results. Rui et al list 3 relevant nomenclature of DOE: i) Factors-the input design parameters to change in the experiment, ii) Levels-the value of the input parameters and iii) Responses-the output associated with design parameters, it is a measure of design performance indicators [20]. Most of author highlight that DoE only used to design initial samples before optimization stage in metamodel optimization problem.

Classic DoE includes full factorial, fractional factorial, central composite design, box-behken and orthogonal array. While, modern DoE improves the classical method such as taguchi, latin hypercube sampling (LHS), Hammersley and monte carlo. Most of the modern DoE method is to fulfill space-filling criteria and improve the optimization of the metamodel. In his case study of effect of LHS in metamodel optimization problem, A. Afzhal et al suggested future research direction of the exploration and exploitation strategies can be assisted by 'adaptive sampling' for metamodel [26]. DoE used for one-shot sampling in experimental design, sequential and adaptive sampling suggest by current researcher for infill sampling sample in computer experiment. Sequential sampling method in metamodel is develop from well-known one-shot DoE method by infill point in sequential manner. In contrast, adaptive sampling method is chosen sample point from previous information of metamodel approximation output. The terms active learning used by Settles (2010) described the approach of selecting the most informative sample points using the metamodel thus it works better even though with fewer points [35]. Due to this, adaptive sampling has the potential to proliferate in research area optimization to refine the model and improve accuracy of metamodel.

\subsection{Infill sampling criterion}

Infill sampling approach for metamodeling is designed to refine the current model by adding sample point. Various of infill criteria developed for adaptive sampling method to meets exploitation, exploration, and balanced exploitation and exploration. There are several infill sampling criterion strategy proposed by previous research such as distance-based design, variance-based design, probability-based design, and Lipchitz based design [15]. Statistical lower bound, the probability of improvement, expected improvement, entropy search, variance-based, lipschitz-based only focused on a statistical model such kriging which is not suitable for radial basis function [36]. This suggested that future research need to enhance drawback of RBF metamodeling method in optimization.

\subsection{Recent method in metamodel infill sampling strategy for RBF metamodeling}

More recent studies finding the best method to obtain new sampling data location as summarize in Table 2. However, the study still under investigation and improve year by year to enhance sampling method using metamodel. Other than that, most of studies in adaptive sampling metamodel optimization method have focused on infill sampling approach. There is still limited study ensemble method to obtaining a new sample as suggested by [37] to develop more effective infill sampling criteria consider more properties of ensembles. Hence, instead of employed ensemble method in metamodel approximation stage, we proposed to implement a consensus method for infill sampling stage method to get the best method of obtaining new sample point. Selection of right model will improve the performance of metamodel.

Historically, research in investigating adaptive sampling or sequential sampling technique surprisingly increases to enhance the performance of the metamodel. In an investigation of sequential using RBF, Havinga et al. conclude that kriging metamodels get better performance when there is no noise is present on the black-box model, whereas when RBF interpolation function with multiquadric activation function performs best if noise is present. RBF metamodels proved to suffer less from fluctuations in global optimization throughout the optimization procedure. Other studies reported that to obtain an RBF metamodel with desirable approximation performance can be divided into two types: (i) enhancing the metamodel itself and (ii) enhancing the sampling techniques [38]. Previous work by Jin et al. (2001) is the one of prominent early study that often cited in research on the development of new sequential sampling approaches for global metamodeling to solve the most issue in sequential sampling. Jin et al. suggested a maximin and crossvalidation strategy with RBF to select current sample points based on location of accessible sample points 
and refine the metamodel [39]. In 2014, Pan developed method extrema points search of metamodels and found minimum points of density function. Taking this integrated approach, he found that his proposed method significantly outperforms than previous work. Theunissen and Gjelstrup proposed another an adaptive sampling method applied point-wise measurement methods to navigate the two-dimensional spatial distribution of sampling point in RBF. The result demonstrates that the new technique tends to be robustness in terms of precision and reliability compared to traditional full-factorial sampling [40]. In a study conducted by Regis and Shoemaker (2005), a proposed novel method to select the next sample points for complex function evaluation that minimizes the current PRS model subject to the constraints and additional constraints that the point is lies of some distance from previously evaluated points [41]. Regis (2011) then suggested a different method called ConstrLMSRBF to construct the RBF metamodel for both the objective function and all the constraint functions in each iteration, these RBF metamodels guided the selection of the next sample points where objective and constraint functions are assessed [42]. In continuous research by Regis (2014a), algorithms designed are called COBRA and extended ConstrLMSRBF, used for highdimensional issues which all initial points located in infeasible design space [43]. Recent studies in 2018 . Wu et al. proposed a new method named RBF-based constrained global optimization (RCGO) to overcome computationally cost objective function and inequality constraints. The method is one of the most practical ways to obtain the initial points for the case study while an auxiliary objective function is designed to identify the next iterative point and improved the metamodel [44]. All the studies discussed, support the hypothesis that using metamodel can improve approximation and process identifying a new sample point could enhance the model's precision by using adaptive sampling technique as shown in Table 1.

Table 1. Summary of recent studies infill sampling strategy

\begin{tabular}{llc}
\hline \multicolumn{1}{c}{ Author } & \multicolumn{1}{c}{ Infill sampling method } & Dimension complex function \\
\hline Guang Pan et al. [45] & Density based & $4^{\text {th }}$ dimensions \\
Cai et al [46] & hybrid with cut- HDMR & $16^{\text {th }}$ dimensions \\
Amouzgar et al [33] & posteriori bias & $16^{\text {th }}$ dimensions \\
Cai et al [47] & Cross validation & $6^{\text {th }}$ dimensions \\
Jin et al [39], Regis and Shoemaker & Distance based & Low dimension \\
[41, 48], Crombeq[49], & & \\
Regis [50], Bajaj et al [51] & Local model trust region & $15^{\text {th }}$ dimensions \\
Zhou [52] & Hybrid with self organizing map & $5^{\text {th }}$ dimension \\
Mackman and Allen [53] & Laplacian criterion & Low dimension \\
Theunissen and Gjelstrup [40] & Pointwise measurement & Low dimension \\
Iuliano [7] & Proper orthogonal decomposition & Low Dimension \\
Khalfallah et al [4] & NGSA II & $8^{\text {th }}$ dimension \\
Wang and Ierapetritou [54] & Error based & $5^{\text {th }}$ and $6^{\text {th }}$ dimension \\
Li et al [55] & Adjusting shape parameter RBF & $20^{\text {th }}$ dimension \\
\hline
\end{tabular}

\section{PROPOSED METHOD}

Based on comprehensive literature studies, the research gap identified infill sampling strategy to obtain new sample point. In this investigation, the method proposed to compare four infill strategy methods that involve distance-based method, hybrid metamodel with deterministic method, hybrid metamodel with metaheuristic method and cross-validation. The proposed method should meet exploitation and exploration in design space. There is a various method of infill sampling criterion to refine the metamodel such as deterministic and stochastic. The deterministic method includes trust region, line search, elimination Lagragarian, etc. Besides, stochastic implementation method for infill sampling strategies such as genetic algorithm, evolutionary algorithm, particle swarm optimization, and evolutionary algorithm. Construction metamodel with a metaheuristic method purposed to outcomes limitation for high dimension problems.

Ensemble method is the way to select the most metamodel perform the best among the model that used. Goel et al identify two (2) benefit of using ensemble in metamodel: i) identify region with high error and ii) robust optimization approach [27]. A study by P. Ye et al implement three metamodel techniques with optimized weight factors is used for the selection of promising sample points, the narrowing of space exploration and the identification of the global optimum [56]. Ensemble method can be classified into non-homogeneous and homogenous method. Non-homogeneous method is referred to agreement of expert or consensus. The proposed design method shows in Figure 2 implement non-homogenous or known as consensus (agreement of expert) for infill sampling strategy. This proposed method is an interesting topic regarding model comparisons is defining which measure of model performance. 


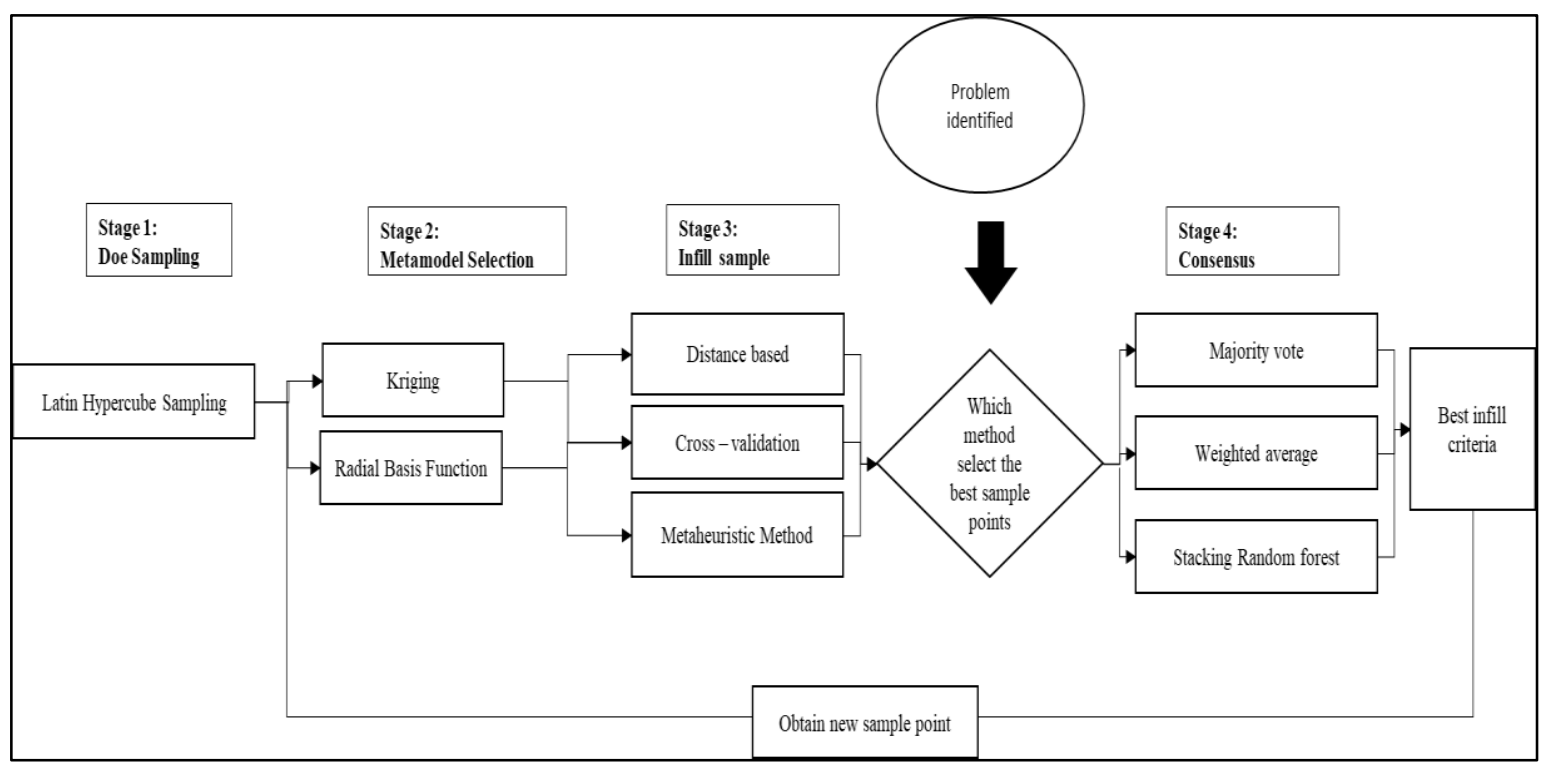

Figure 2. Proposes method of metamodel adaptive sampling using consensus

\section{CONCLUSION AND FUTURE RESEARCH PROSPECT}

The most challenge issue of the metamodel when area of the design space, the computational demand, and the required number of points exponentially increase with dimensions of the input variables which is known as the "curse of dimensionality". The proposed method using a consensus of metamodels can improve robustness of the predictions sample location based on best model voting. Current studies, implementing ensemble method in approximation stage to choose the best metamodel before algorithm allows to select sample point and refine the metamodel. Comparative study between kriging and RBF shows that kriging highly outperforms for low design variable while RBF achieves better performance in high design variables. Future research direction for adaptive sampling in metamodel should consider impact of kernel function, multi-objective optimization, and employed multiple infill sampling for each iteration.

\section{ACKNOWLEDGEMENTS}

We would like to express our gratitude to Malaysia-Japan International Institute of Technology (MJIT), Universiti Teknologi Malaysia (UTM) for their financial support.

\section{REFERENCES}

[1] R. Ghiasi, M. R. Ghasemi, and M. Noori, "Comparative studies of metamodeling and AI-Based techniques in damage detection of structures," Advances in Engineering Software, vol. 125, pp. 101-112, Nov 2018.

[2] R. P. Liem, C. A. Mader, and J. R. R. A. Martins, "Surrogate models and mixtures of experts in aerodynamic performance prediction for aircraft mission analysis," Aerospace Science and Technology, vol. 43, pp. 126-151, 2015.

[3] T. Wortmann, A. Costa, G. Nannicini, and T. Schroepfer, "Advantages of surrogate models for architectural design optimization," Artificial Intelligence for Engineering Design, Analysis and Manufacturing AIEDAM, vol. 29, no. 4, pp. 471-481, 2015.

[4] S. Khalfallah and A. Ghenaiet, "Radial basis function-based shape optimization of centrifugal impeller using sequential sampling," Proceedings of the Institution of Mechanical Engineers, Part G: Journal of Aerospace Engineering, vol. 229, no. 4, pp. 648-665, 2015.

[5] A. I. J. Forrester and A. J. Keane, "Recent advances in surrogate-based optimization," Progress in Aerospace Sciences, vol. 45, no. 1-3, pp. 50-79, Jan-April 2009.

[6] H. Dong, B. Song, Z. Dong, and P. Wang, "SCGOSR: Surrogate-based constrained global optimization using space reduction," Applied Soft Computing J, vol. 65, pp. 462-477, April 2018.

[7] E. Iuliano, "Adaptive Sampling Strategies for Surrogate-based Aerodynamic Optimization," Springer Tracts Mech. Eng, pp. 25-46, Jan 2016

[8] E. Iuliano, "Global optimization of benchmark aerodynamic cases using physics-based surrogate models," Aerospace Science and Technology, vol. 67, pp. 273-286, August 2017.

[9] S. ur Rehman and M. Langelaar, "Expected improvement based infill sampling for global robust optimization of constrained problems," Optimization and Engineering, vol. 18, no. 3, pp. 723-753, 2017. 
[10] P. N. K. and J. K. A. T. W. Simpson1, J. D. Peplinski, "Metamodels for Computer-based Engineering Design: Survey and recommendations," Engineering with Computers, vol. 17, no. 1, pp. 129-150, 2001.

[11] G. G. Wang and S. Shan, "Review of Metamodeling Techniques in Support of Engineering Design Optimization," Journal of Mechanical Design-J MECH DESIGN, vol. 129, no. 4, p. 370, 2007.

[12] S. Shan and G. G. Wang, "Survey of modeling and optimization strategies to solve high-dimensional design problems with computationally-expensive black-box functions," Structural and Multidisciplinary Optimization, vol. 41, no. 2, pp. 219-241, 2010.

[13] N. V. Queipo, R. T. Haftka, W. Shyy, T. Goel, R. Vaidyanathan, and P. Kevin Tucker, "Surrogate-based analysis and optimization," Progress in Aerospace Sciences, vol. 41, no. 1, pp. 1-28, 2005.

[14] A. Bhosekar and M. Ierapetritou, "Advances in surrogate based modeling, feasibility analysis, and optimization: A review," Computer \& Chemical Engineering, vol. 108, pp. 250-267, Jan 2018.

[15] H. Liu, Y. Ong, and J. Cai, "A Survey of Adaptive Sampling for Global Metamodeling in Support of Simulationbased Complex Engineering Design A survey of adaptive sampling for global metamodeling in support of simulation-based complex engineering design," Structural and Multidisciplinary Optimization, vol. 57, pp. 393-416, 2018.

[16] J. P. C. Kleijnen, "Kriging metamodeling in simulation: A review," European Journal of Operational Research, vol. 192, no. 3, pp. 707-716, Feb 2009.

[17] N. Qiu, G. Sun, Q. Li, J. Fang, and N. H. Kim, "On design optimization for structural crashworthiness and its state of the art," Structural and Multidisciplinary Optimization, vol. 55, no. 3, pp. 1091-1119, 2017.

[18] R. Yondo, E. Andrés, and E. Valero, "A review on design of experiments and surrogate models in aircraft real-time and many-query aerodynamic analyses," Progress in Aerospace Sciences, vol. 96, no. 2017, pp. 23-61, 2018.

[19] T. Chatterjee, S. Chakraborty, and R. Chowdhury, "A Critical Review of Surrogate Assisted Robust Design Optimization," Archives of Computational Methods in Engineering, vol. 26, no. 1, pp. 245-274, 2019.

[20] R. Xi, H. Jia, and Q. Xiao, "Study of experimental design and Response Surface method for surrogate model of computational simulation," 2011 International Conference on Electrical and Control Engineering, Yichang, pp. 4995-4998, 2011.

[21] T. Østergård, R. L. Jensen, and S. E. Maagaard, "A comparison of six metamodeling techniques applied to building performance simulations," Applied Energy, vol. 211, pp. 89-103, Feb 2018.

[22] P. Chaudhury and H. K. Tripathy, "Optimising the parameters of a RBFN network for a teaching learning paradigm," Indonesian Journal of Electrical Engineering and Computer Science IJEECS, vol. 15, no. 1, pp. 435-442, 2019.

[23] M. Nafea, A. R. M. Ali, J. Baliah, and M. S. M. Ali, "Metamodel-based optimization of a PID controller parameters for a coupled-tank system," Telecommunication, Computing, Electronics and Control, TELKOMNIKA, vol. 16, no. 4, pp. 1590-1596, 2018.

[24] M. Arian Nik, K. Fayazbakhsh, D. Pasini, and L. Lessard, "A comparative study of metamodeling methods for the design optimization of variable stiffness composites," Composite Structures, vol. 107, pp. 494-501, Jan 2014.

[25] G. Vicario, G. Craparotta, and G. Pistone, "Meta-models in Computer Experiments: Kriging versus Artificial Neural Networks," Quality and Reliability Engineering International, vol. 32, no. 6, pp. 2055-2065, June 2016.

[26] A. Afzal, K. Y. Kim, and J. W. Seo, "Effects of latin hypercube sampling on surrogate modeling and optimization," International Journal of Fluid Machinery and System, vol. 10, no. 3, pp. 240-253, 2017.

[27] T. Goel, R. T. Haftka, W. Shyy, and N. V. Queipo, "Ensemble of surrogates," Structural and Multidisciplinary Optimization, vol. 33, no. 3, pp. 199-216, 2007.

[28] Q. Zhou, X. Shao, P. Jiang, Z. Gao, H. Zhou, and L. Shu, "An active learning variable-fidelity metamodelling approach based on ensemble of metamodels and objective-oriented sequential sampling," Journal of Engineering Design, vol. 27, no. 4-6, pp. 205-231, 2016.

[29] S. A. G. Shirazi and M. B. Menhaj, "A new genetic based algorithm for channel assignment problems," Computational Intelligence, Theory and Applications, pp. 85-91, 2006.

[30] A. Ghasempour, "Using a genetic-based algorithm to solve the scheduling optimization problem for long-range molecular communications in nanonetworks," 2015 IEEE 26th Annual International Symposium on Personal, Indoor, and Mobile Radio Communications PIMRC, Hong Kong, pp. 1825-1829, 2015.

[31] F. Li, X. Cai, and L. Gao, "Ensemble of surrogates assisted particle swarm optimization of medium scale expensive problems," Applied Soft Computing, vol. 74, pp. 291-305, Jan 2019.

[32] M. Cavazzuti, "Optimization Methods: From Theory to Design Scientific and Technological Aspects in Mechanics," Springer Berlin Heidelberg, 2012.

[33] K. Amouzgar and N. Strömberg, "Radial basis functions as surrogate models with a priori bias in comparison with a posteriori bias," Structural and Multidisciplinary Optimization, vol. 55, no. 4, pp. 1453-1469, 2017.

[34] T. R. Saeed, J. Salman, and A. H. Ali, "Classification improvement of spoken arabic language based on radial basis function," International Journal of Electrical and Computer Engineering IJECE, vol. 9, no. 1, pp. 402-408, 2019.

[35] B. Settles, “Active Learning Literature Survey," Computer Sciences Technical Report 1648, Jan 2009.

[36] W. Yao, X. Q. Chen, Y. Y. Huang, and M. Van Tooren, "A surrogate-based optimization method with RBF neural network enhanced by linear interpolation and hybrid infill strategy," Optimization Methods and Software, vol. 29, no. 2, pp. 406-429, 2014.

[37] D. Guo, Y. Jin, J. Ding and T. Chai, "Heterogeneous Ensemble-Based Infill Criterion for Evolutionary Multiobjective Optimization of Expensive Problems," in IEEE Transactions on Cybernetics, vol. 49, no. 3, pp. 1012-1025, March 2019. 
[38] J. Havinga, A. H. van den Boogaard, and G. Klaseboer, "Sequential improvement for robust optimization using an uncertainty measure for radial basis functions," Structural and Multidisciplinary Optimization, vol. 55, no. 4, pp. 1345-1363, 2017.

[39] R. Jin, W. Chen, and A. Sudjianto, "On Sequential Sampling for Global Metamodeling in Engineering Design," Proceedings of the ASME Design Engineering Technical Conferencepp, pp. 539-548, 2002.

[40] R. Theunissen and P. Gjelstrup, "Adaptive sampling in higher dimensions for point-wise experimental measurement techniques," Measurement Science and Technology, vol. 29, no. 8, 2018.

[41] R. G. Regis and C. A. Shoemaker, "Constrained global optimization of expensive black box functions using radial basis functions," Journal of Global Optimization, vol. 31, no. 1, pp. 153-171, 2005.

[42] R. G. Regis, "Stochastic radial basis function algorithms for large-scale optimization involving expensive blackbox objective and constraint functions," Computer \& Operations Research, vol. 38, no. 5, pp. 837-853, May 2011.

[43] R. G. Regis, "Constrained optimization by radial basis function interpolation for high-dimensional expensive blackbox problems with infeasible initial points," Journal Engineering Optimization, vol. 46, no. 2, pp. 218-243, 2014.

[44] Y. Wu, Q. Yin, H. Jie, B. Wang, and J. Zhao, "A RBF-based constrained global optimization algorithm for problems with computationally expensive objective and constraints," Structural and Multidisciplinary Optimization, vol. 58, no. 4, pp. 1633-1655, 2018.

[45] G. Pan, P. Ye, P. Wang, and Z. Yang, "A sequential optimization sampling method for metamodels with radial basis functions," The Scientific World Journal, vol. 2014, 2014.

[46] X. Cai, H. Qiu, L. Gao, P. Yang, and X. Shao, "An enhanced RBF-HDMR integrated with an adaptive sampling method for approximating high dimensional problems in engineering design," Structural and Multidisciplinary Optimization, vol. 53, no. 6, pp. 1209-1229, 2016.

[47] X. Cai, H. Qiu, L. Gao, L. Wei, and X. Shao, "Adaptive Radial-Basis-Function-Based Multifidelity Metamodeling for Expensive Black-Box Problems,” AIAA J, vol. 55, no. 7, pp. 2424-2436, 2017.

[48] R. G. Regis and C. A. Shoemaker, "Improved strategies for radial basis function methods for global optimization," Journal of Global Optimization, vol. 37, no. 1, pp. 113-135, 2007.

[49] K. Crombecq, L. De Tommasi, D. Gorissen and T. Dhaene, "A novel sequential design strategy for global surrogate modeling," Proceedings of the 2009 Winter Simulation Conference WSC, Austin, TX, USA, pp. 731-742, 2009.

[50] R. G. Regis and C. A. Shoemaker, "Parallel radial basis function methods for the global optimization of expensive functions," European Journal of Operational Research, vol. 182, no. 2, pp. 514-535, Oct 2007.

[51] I. Bajaj, S. S. Iyer, and M. M. Faruque Hasan, "A trust region-based two phase algorithm for constrained black-box and grey-box optimization with infeasible initial point," Computers \& Chemical Engineering, vol. 116, pp. 306-321, August 2018.

[52] Q. Zhou et al., "An active learning radial basis function modeling method based on self-organization maps for simulation-based design problems," Knowledge-Based Systems, vol. 131, pp. 10-27, Sep 2017.

[53] T. J. Mackman and C. B. Allen, "Investigation of an adaptive sampling method for data interpolation using radial basis functions," International Journal for Numerical Methods in Engineering, no. March, pp. 915-938, 2010.

[54] M. Wang, Z., Ierapetritou, "A novel feasibility analysis method for black-box processes using a radial basis function adaptive sampling approach," AICHE Journal, vol. 55, no. 7, pp. 2424-2436, 2017.

[55] G. Li, Q. Zhang, J. Sun and Z. Han, "Radial Basis Function Assisted Optimization Method with Batch Infill Sampling Criterion for Expensive Optimization," 2019 IEEE Congress on Evolutionary Computation (CEC), Wellington, New Zealand, pp. 1664-1671, 2019.

[56] P. Ye, G. Pan, and Z. Dong, "Ensemble of surrogate based global optimization methods using hierarchical design space reduction," Structural and Multidisciplinary Optimization, vol. 58, no. 2, pp. 537-554, 2018.

\section{BIOGRAPHIES OF AUTHORS}

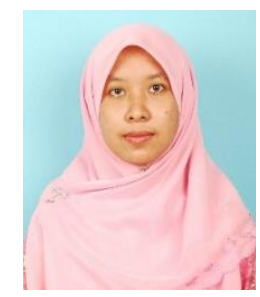

\section{Che Munira Che Razali}

Department of Electronics System Engineering

Malaysia-Japan International Institute of Technology

Universiti Teknologi Malaysia

$\mathrm{PhD}$ Student

Optimization, Metamodel, Machine Learning

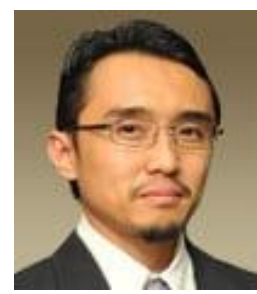

Assoc. Prof. Dr Shahrum Shah Abdullah

Department of Electronics System Engineering

Malaysia-Japan International Institute of Technology

Universiti Teknologi Malaysia

Associate Professor

Intelligent control, Artificial Intelligence, Industrial Process Control 


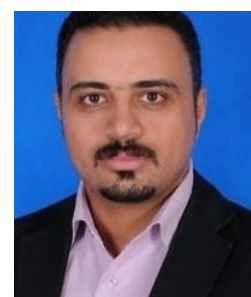

Dr Amir Parnianifard

Department of Mechanical and Manufacturing Engineering,

Faculty of Engineering,

Universiti Putra Malaysia

Post-Doctoral Kharazmi University, Iran

Industrial Engineering, Metamodel, Sensitivity Analysis

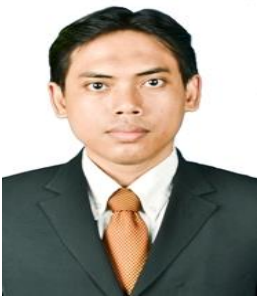

\section{Amrul Farul}

Department of Electronics System Engineering

Malaysia-Japan International Institute of Technology

Universiti Teknologi Malaysia

PhD Student

Machine Learning, Flood forecasting, Optimization 